\title{
Eficiência climática para as culturas da soja e do trigo no estado do Rio Grande do Sul em diferentes datas de semeadura
}

\author{
Climatic efficiency for soybean and wheat crops in the state of Rio Grande do Sul, Brazil, in \\ different sowing date
}

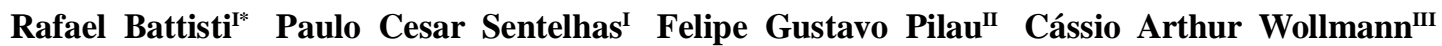

\section{RESUMO}

Eficiência climática (EC) é um índice que demonstra em termos quantitativos a redução produtiva ocasionada pelo déficit hídrico. Assim, este trabalho teve como objetivo avaliar a EC das culturas da soja e do trigo para o estado do Rio Grande do Sul, considerando-se diferentes locais e datas de semeaduras. A EC foi obtida pela relação entre as produtividades atingível (PA) e potencial (PPf), estimadas por meio de modelos de simulação. A PA foi obtida pela estimativa da PPf, a qual foi determinada pelo método de Zona Agroecológica da FAO, deflacionada pelo déficit hidrico em cada uma das fases das culturas para cada data de semeadura, entre os anos de 1979 e 2008. Os resultados evidenciaram que as datas de semeadura influenciam a EC para a cultura da soja, a qual variou de 0,31 a 0,61. Com base nos resultados de EC e PA, há um ganho de produtividade com o atraso da semeadura de 01/10 para 21/12. Para a cultura do trigo, a EC média foi superior a 0,81, o que leva a concluir que se deve optar por datas de semeadura em que há maior PPf. As maiores PPf para o trigo são obtidas nas semeaduras a partir de meados do mês de junho ao final do mês de julho.

Palavras-chave: deficiência hídrica, método zona agroecológica, época de semeadura.

\section{ABSTRACT}

Climatic efficiency (EC) is an index that shows the yield loss caused by water deficiency. The objective of this study was to evaluate the EC of soybean and wheat crops for the state of Rio Grande do Sul, Brazil. The EC was obtained by the ratio between the attainable yield (PA) and potential yield (PPf) of these crops, estimated by crop simulation models. The PA was obtained by estimating the potential yield (PPf), which was determined by the FAO Agroecological Zone method, depreciated by the water deficit in each crop phenological phase for each sowing date, between 1979 and 2008. The results showed that sowing dates influenced the EC for the soybean crop, ranging from 0.31 to 0.61. Based on the results from EC and PA, there is an increase of crop yield when sowings are delayed from 01/10 to 21/12. For the wheat crop, the average EC was greater than 0.81, which allows to conclude that the best sowing dates should be chosen considering the highest PPf. The highest potential yields for the wheat crop were obtained for sowings from mid-June to end July.

Key words: water deficiency, agroecological zone method, sowing dates.

\section{INTRODUÇÃO}

As culturas da soja e do trigo são de grande importância econômica para o Brasil e principalmente para o estado do Rio Grande do Sul. A precipitação pluvial durante a safra da soja determina grande parte da variabilidade interanual da produção, estima-se que, para a soja, $93 \%$ das perdas na safra ocorram em razão do déficit hídrico (BERLATO \& FONTANA, 2003). Para a cultura do trigo, o déficit hídrico também resulta em perdas de produtividade, mas com eventos menos frequentes, quando comparados aos que ocorrem na cultura da soja (ALBERTO et al., 2006).

Sendo a precipitação pluvial a principal variável meteorológica a afetar a produtividade das culturas agrícolas de sequeiro, podem-se empregar índices agroclimáticos para auxiliar na escolha de

\footnotetext{
'Departamento de Engenharia de Biossistemas, Escola Superior de Agricultura “Luiz de Queiroz" (ESALQ), Universidade de São Paulo (USP), CP 09, 13418-900, Piracicaba, SP, Brasil. E-mail: r.battisti@ hotmail.com. *Autor para correspondência.

IIDepartamento de Fitotecnia, Universidade Federal de Santa Maria (UFSM), Santa Maria, RS, Brasil.

IIIDepartamento de Geociências, UFSM, Santa Maria, RS, Brasil.
} 
datas preferenciais de semeadura. Dentre esses índices, o de Satisfação das Necessidades de Água (ISNA), que expressa a relação entre as evapotranspirações real e a máxima da cultura, durante as fases críticas das culturas, vem sendo utilizado na elaboração do zoneamento de risco climático, o qual delimita áreas e períodos de semeadura favoráveis ao cultivo de culturas agrícolas no Brasil, desenvolvido pelo Ministério da Agricultura, Pecuária e Abastecimento (MAPA, 2012). Já o índice de eficiência climática (EC), obtido pela relação entre as produtividades atingível e potencial, demonstra, em termos quantitativos, a redução produtiva ocasionada pelo déficit hídrico. $\mathrm{O}$ índice de $\mathrm{EC}$ foi utilizado por BONNECARRÈRE et al. (2007) para a cultura do milho em diferentes localidades do Rio Grande do Sul para identificar datas preferenciais de semeadura. Considerando-se que o uso de modelos de simulação para a determinação da EC é um meio eficiente para se determinar os riscos climáticos associados às culturas agrícolas, o presente estudo teve por objetivo estimar a EC para as culturas da soja e do trigo, em diferentes datas de semeaduras para o estado do Rio Grande do Sul, visando a identificar locais e datas preferenciais para semeadura dessas culturas, minimizando assim o risco de perdas por déficit hídrico.

\section{MATERIAL E MÉTODOS}

Os dados meteorológicos empregados no presente estudo correspondem ao período entre 1979 e 2008, organizados na escala decêndial, contemplando precipitação pluvial, temperatura média do ar, insolação média diária, fotoperíodo médio e radiação solar global extraterrestre média, os quais foram obtidos junto às seguintes fontes: Agência Nacional de Águas; Fundação Estadual de Pesquisa Agropecuária do Rio Grande do Sul; e Empresa Brasileira de Pesquisa Agropecuária, para as seguintes localidades do estado do Rio Grande do Sul: Encruzilhada do Sul (30³1'S, 52 $31^{\circ}$ 'W, 432m);

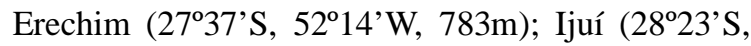
535' 'W, 328m); Júlio de Castilhos (29¹3'S,

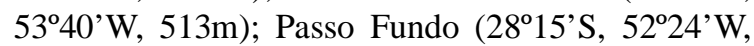

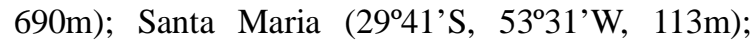
Santa Rosa (27 $\left.52^{\prime} \mathrm{S}, 5^{\circ} 28^{\prime} \mathrm{W}, 227 \mathrm{~m}\right)$; São Borja

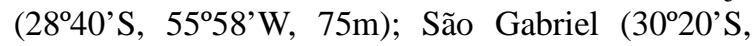

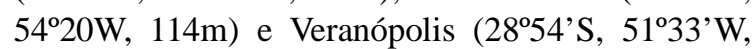
$705 \mathrm{~m}$ ), representando diferentes regiões produtoras de soja e trigo do estado gaúcho.

Para a estimativa da produtividade potencial (PPf), empregou-se o método da Zona
Agroecológica descrito por DOORENBOS \& KASSAM (1994), obtido pela equação: $P P f=(P P B p *$ $\left.\mathrm{C}_{\mathrm{IAF}} * \mathrm{C}_{\mathrm{R}} * \mathrm{C}_{\mathrm{C}} * \mathrm{ND}\right) /(1-0,01 * \mathrm{U} \%)$, em que PPBp é a produtividade potencial bruta padrão de matéria seca em $\mathrm{kg} \mathrm{ha}^{-1} \mathrm{dia}^{-1}$ de uma cultura padrão com índice de área foliar igual a 5, $\mathrm{C}_{\mathrm{IAF}}$ é a correção para índice de área foliar, $\mathrm{C}_{\mathrm{R}}$ é a correção para a taxa de respiração, $\mathrm{C}_{\mathrm{C}}$ é a correção para a parte colhida, ND é o número de dias do ciclo de produção e U\% é a umidade residual na parte colhida.

A PPBp foi estimada para cada decêndio e acumulada ao longo do ciclo. A PPBp foi obtida pela soma das produtividades potenciais padrão para período de céu limpo (PPBc) e de céu nublado (PPBn), obtidas por: $\mathrm{PPBc}=[(107,2+8,604 * \mathrm{Qo}) * \mathrm{cTc} * \mathrm{n} / \mathrm{N}]$ e $\left.\mathrm{PPBn}=[(31,7+5,234 * \mathrm{Q}))^{*} \mathrm{cTn}{ }^{*}(1-\mathrm{n} / \mathrm{N})\right]$, em que Qo é a radiação solar global extraterrestre em $\mathrm{MJ} \mathrm{m}^{-2} \mathrm{~d}^{-1}$, cTc e cTn são fatores de correção para temperatura do ar para dia de céu claro e nublado (PEREIRA et al., 2002), n é a insolação diária em horas por dia e $\mathrm{N}$ é o número de horas máximo de brilho solar diário (fotoperíodo).

Para o $C_{R}$, adotou-se o valor de 0,6 para os decêndios em que a temperatura média do ar foi menor que $20^{\circ} \mathrm{C}$ e de 0,5 para os decêndios em que a temperatura média do ar foi maior ou igual a $20^{\circ} \mathrm{C}$. Os coeficientes de colheita $(\mathrm{Cc})$ adotados foram iguais a 0,35 e 0,4 e as umidades residuais (U\%) iguais a 13 e $13,5 \%$, respectivamente, para as culturas da soja e do trigo (DOORENBOS \& KASSAM, 1994). O número de dias do ciclo (ND) para a soja foi de 140 dias (CUNHA et al., 2001a), enquanto que para o trigo foi de 130 dias (OSÓRIO \& WENDT, 1995). Para o $C_{\mathrm{IAF}}$, adotou-se como índice de área foliar máximo $\left(\mathrm{IAF}_{\max }\right)$ para a soja o valor de 4,5 (RODRIGUES et al., 2006) e para o trigo de 4 (MOREIRA et al., 1999), obtendo o $\mathrm{C}_{\mathrm{IAF}}$ pela equação: $\mathrm{C}_{\mathrm{IAF}}=\left(0,0093+0,185 * \mathrm{IAF}_{\max }{ }^{-}\right.$ $\left.0,0175 * \mathrm{IAF}_{\max }^{2}\right)$.

Para a estimativa da produtividade atingível (PA), considerou-se a penalização da PPf pelo déficit hídrico, sendo que, para a primeira fase de desenvolvimento da cultura, o valor de PPf correspondeu ao total acumulado no ciclo da cultura, já, para a fase seguinte, esse valor correspondeu à PA obtida ao final da fase anterior, assim como para as fases subsequentes, na forma de um produtório, pela equação: $\left.\mathrm{PA}=\prod\left\{1-\mathrm{ky}_{\mathrm{i}}(1-\mathrm{ETr} / \mathrm{ETc})\right)^{*} \mathrm{PPf}\right\}$, em que ky é o coeficiente de sensibilidade ao déficit hídrico para cada fase de desenvolvimento (i) da cultura, ou seja, das fases vegetativa, reprodutiva e de maturação. Os valores de ky adotados para essas três fases foram de 0,2, 0,8 e 1,0 para a soja e de 0,2,0,6 e 0,0 para o trigo (DOORENBOS \& KASSAM, 1994). 
A evapotranspiração da cultura (ETc) foi obtida pelo produto entre a evapotranspiração de referência (ETo), estimada pelo método de Camargo (PEREIRA et al., 2002), e o coeficiente de cultura (kc) para cada fase das culturas. Os valores de kc adotados foram iguais a 0,$5 ; 1,15$ e 0,5 para a cultura da soja (BERLATO et al., 1986) e iguais a 0,7, 0,9 e 0,65 para a cultura do trigo (CUNHA et al., 2001b), respectivamente, para as fases vegetativa, reprodutiva e de maturação. Quanto à evapotranspiração real (ETr), seguiram-se os critérios do balanço hídrico de THORNTHWAITE \& MATHER (1955) para sua determinação, adotando-se uma capacidade de água disponível no solo de 50mm (CUNHA et al., 2001a), considerando-se que não havia restrição hídrica no solo para o estabelecimento da cultura.

Quanto à eficiência climática (EC), esta foi obtida pela relação entre a PA e a PPf, simulandose semeaduras dentro do período recomendado pelo zoneamento agrícola, sendo: 01/10; 11/10; 21/10; $01 / 11 ; 11 / 11 ; 21 / 11 ; 01 / 12 ; 11 / 12$ e $21 / 12$, para a cultura da soja (CUNHA et al., 2001a), e 01/05; $11 / 05$; 21/05; 01/06; 11/06; 21/06; 01/07; 11/21 e 21/07, para a cultura do trigo (CUNHA et al., 2001b).

Com as estimativas da PPf, da PA e da

EC, realizou-se a análise de variância com base em dois fatores, um quantitativo, referente às datas de semeadura e outro qualitativo, referente à localidade de cultivo. Havendo diferença significativa das variáveis citadas entre datas de semeadura, realizouse a análise de regressão entre essas e o número de dias após $01 / 05$ para a cultura do trigo e 01/10 para a cultura da soja, respectivamente, com base na média de cada decêndio para cada local. Posteriormente, havendo diferença entre localidades, realizou-se o teste complementar de Duncan (5\%) para se comparar as médias de cada local. Havendo interação entre data de semeadura e local de cultivo, procedeu-se à análise de regressão em função da data de semeadura, isolando-se tal fator para cada localidade.

\section{RESULTADOS E DISCUSSÃO}

Os resultados na análise de variância apresentaram nível de significância de $1 \%$ para as variáveis PPf, PA e EC ao avaliar o fator local e data de semeadura de forma isolada para a cultura da soja e do trigo (Tabela 1). Não se observou interação entre época de semeadura e local de cultivo. Assim, com base nos resultados apresentados, verifica-se que, para as variáveis analisadas, a época de semeadura influencia no resultado de forma igual para todas as localidades, mas há diferença significativa entre localidades para PPf, PA e EC.

Para a cultura da soja, a PPf alcançou valor médio máximo de $6.887 \mathrm{~kg} \mathrm{ha}^{-1}$ para o município de São Borja, diferindo significativamente dos demais, enquanto que o menor valor médio foi obtido em Encruzilhada do Sul, com produtividade de $6.080 \mathrm{~kg}$ $\mathrm{ha}^{-1}$, sendo estatisticamente igual ao município de Veranópolis (Tabela 2). VENTIMIGLIA et al. (1999), avaliando a PPf da soja em condições irrigadas em Eldorado do Sul, RS, obteve PPf de $10.000 \mathrm{~kg} \mathrm{ha}^{-1}$, esta estimada em função do número de legumes presentes na planta no estádio R5, enquanto que a PPf final observada foi de $4.600 \mathrm{~kg} \mathrm{ha}^{-1}$ de grãos, redução associada às perdas referentes às condições climáticas, disponibilidade nutricional e estresses bióticos, que reduziram a capacidade de retenção de legumes.

Ao se relacionar os valores de PPf em função de dias após 01/10 para a cultura da soja, obteve-se uma equação quadrática (Figura 1a), a qual demonstra que, para o intervalo de semeadura entre 01/10 e 21/12, a data com maior PPf ocorre

Tabela 1 - Nível de significância de p para análise de variância para o fator local (L), data de semeadura (DS) e a interação L x DS para a produtividade potencial (PPf), produtividade atingível (PA) e eficiência climática (EC) e os respectivos coeficiente de variação (CV) para as culturas da soja e do trigo.

\begin{tabular}{ccccc}
\hline & Variável & Local (L) & Data de semeadura (DS) & L X DS \\
\hline \multirow{3}{*}{ Soja } & PPf & $<0,0001$ & $<0,0001$ & 0,9918 \\
& IPA & $<0,0001$ & $<0,0001$ & 0,9641 \\
& IEC & $<0,0001$ & $<0,0001$ & 0,9848 \\
Trigo & & & & 41 \\
& PPf & $<0,0001$ & $<0,0001$ & 0,0802 \\
& PA & $<0,0001$ & $<0,0001$ & 0,9999 \\
\hline
\end{tabular}

${ }^{1}$ dados transformados por raiz quadrada para análise de variância.

Ciência Rural, v.43, n.3, mar, 2013. 
Tabela 2 - Produtividade potencial ( $\mathrm{PPf}, \mathrm{Kg} \mathrm{ha}^{-1}$ ), produtividade atingivel ( $\mathrm{PA}, \mathrm{kg} \mathrm{ha}^{-1}$ ) e eficiència climática (EC) para as culturas da soja $\mathrm{e}$ do trigo para diferentes municípios do Rio Grande do Sul, com base na média de todos os decêndios de semeadura, para o período de 1979 a 2008 .

\begin{tabular}{|c|c|c|c|c|c|c|}
\hline \multirow{2}{*}{ Local } & \multirow[b]{2}{*}{ PPf } & \multirow[b]{2}{*}{ PA } & \multirow[b]{2}{*}{$\mathrm{EC}$} & \multirow[b]{2}{*}{ PPf } & \multirow[b]{2}{*}{$\mathrm{PA}$} & \multirow[b]{2}{*}{$\mathrm{EC}$} \\
\hline & & & & & & \\
\hline Enc. do Sul & $6.080 \mathrm{~g}$ & $2.292 \mathrm{~d}$ & $0,38 \mathrm{de}$ & $4.107 \mathrm{e}$ & $3.661 \mathrm{~cd}$ & $0,89 a b c$ \\
\hline Erechim & $6.193 \mathrm{ef}$ & $3.776 \mathrm{a}$ & $0,61 \mathrm{a}$ & $4.478 \mathrm{a}$ & $4.109 a$ & $0,92 \mathbf{a}$ \\
\hline Ijuí & $6.469 \mathrm{c}$ & $2.780 \mathrm{c}$ & $0,43 \mathrm{dc}$ & $4.368 \mathrm{bc}$ & $3.715 \mathrm{c}$ & $0,85 \mathrm{e}$ \\
\hline J. de Castilhos & $6.529 \mathrm{c}$ & $2.886 \mathrm{c}$ & $0,45 \mathrm{c}$ & $4.393 \mathrm{ab}$ & $3.938 \mathrm{~b}$ & $0,90 \mathrm{ab}$ \\
\hline Passo Fundo & $6.225 \mathrm{ed}$ & $3.447 \mathrm{~b}$ & $0,56 \mathrm{~b}$ & $4.295 \mathrm{~cd}$ & $3.940 \mathrm{~b}$ & $0,92 \mathbf{a}$ \\
\hline Santa Maria & $6.289 \mathrm{~d}$ & $2.317 \mathrm{~d}$ & $0,38 \mathrm{e}$ & $4.167 \mathrm{e}$ & $3.619 \mathrm{~cd}$ & $0,87 \mathrm{de}$ \\
\hline Santa Rosa & $6.491 \mathrm{c}$ & $2.816 \mathrm{c}$ & $0,44 \mathrm{c}$ & $4.276 \mathrm{~cd}$ & $3.709 \mathrm{c}$ & $0,87 \mathrm{de}$ \\
\hline São Borja & $6.887 \mathrm{a}$ & $2.501 \mathrm{~d}$ & $0,37 \mathrm{e}$ & $4.401 \mathrm{ab}$ & $3.569 \mathrm{~d}$ & $0,81 \mathbf{f}$ \\
\hline São Gabriel & $6.638 \mathrm{~b}$ & $1.977 \mathrm{e}$ & $0,31 \mathbf{f}$ & $4.260 \mathrm{~d}$ & $3.711 \mathrm{c}$ & $0,87 \mathrm{cde}$ \\
\hline Veranópolis & $6.139 \mathrm{gf}$ & $3.134 b$ & $0,52 \mathrm{~b}$ & $4.465 \mathrm{ab}$ & $3.963 \mathrm{~b}$ & $0,88 \mathrm{bcd}$ \\
\hline
\end{tabular}

no dia $16 / 10$, a partir de quando ocorre redução gradativa até o período final da janela de semeadura. BONNECARRÈRE et al. (2007), para a cultura do milho, realizaram simulações de semeadura entre 01/10 e 21/12 para diferentes municípios do Rio Grande do Sul e obtiveram resultados similares em que a maior PPf foi obtida nas primeiras épocas de semeadura, tendo uma queda nos períodos posteriores.

Com relação à produtividade atingível (PA) para a cultura da soja, pode-se verificar que há uma maior variação entre as localidades, quando comparada com a PPf (Tabela 2), variação que também é observada para as épocas de semeadura, devido ao menor valor de $\mathrm{r}^{2}$ das equações ajustadas (Figura 1c). Para os municípios, Erechim foi o que apresentou a maior PA, com resultado médio de $3776 \mathrm{~kg} \mathrm{ha}^{-1}$, diferindo estatisticamente das demais localidades. O pior desempenho foi obtido em São Gabriel, com PA de $1977 \mathrm{~kg} \mathrm{ha}^{-1}$, apesar de essa localidade ter apresentado uma das maiores PPf (Tabela 2). Tais resultados demonstram que a precipitação pluvial, principal variável meteorológica que afeta a PA, apresenta variação entre os locais de cultivo para a cultura da soja.

Os valores de PA obtidos no presente estudo se assemelham aos dados médios de produtividade, observados (PO) pelo Instituto Brasileiro de Geografia e Estatística (IBGE, 2010), que, entre 1990 a 2008, variaram de 666 a $2.400 \mathrm{~kg} \mathrm{ha}^{-1}$ para o estado do Rio Grande do Sul. Deve-se ressaltar que a PA considera apenas perdas por déficit hídrico. Desse modo, valores de PA tendem a ser maiores do que os da PO apresentados pelo IBGE, já que outros fatores associados ao manejo da cultura não são considerados pelo modelo de simulação utilizado. A relação entre
PO e PA é denominada de eficiência agrícola (EA), a qual indica o nível tecnológico adotado na condução da cultura. A EA para esse mesmo conjunto de dados foi determinada por BATTISTI et al. (2012).

Para a PA para a cultura da soja, obtevese ajuste linear, demonstrando que, com o atraso da semeadura de 01/10 até 21/12, há um aumento da produtividade de $7,10 \mathrm{~kg} \mathrm{ha}^{-1} \mathrm{dia}^{-1}$ (Figura 1c). BERGAMASCHI et al. (2007) comentaram que a distribuição irregular das chuvas durante o ciclo da cultura da soja pode explicar uma série de atributos de rendimento sob as condições de cultivo de sequeiro. Tal fato deve ser observado ao se escolher as datas preferenciais de semeadura, optando-se sempre que possível por aquelas de maior eficiência climática (EC).

A EC permite identificar as datas de semeadura em que há menores perdas de produtividade por déficit hídrico. Para a cultura da soja, observouse que Erechim obteve a maior EC média $(0,61)$, ou seja, há uma perda de $39 \%$ na PPf devido ao déficit hídrico, diferindo das demais localidades (Tabela 2). Em seguida, vêm as localidades de Passo Fundo e Veranópolis com EC média de 0,56 e 0,52, respectivamente (Tabela 2). A menor EC foi obtida em São Gabriel, com 0,31, demonstrando que, para esse município, estratégias de irrigação podem ser utilizadas para aumentar a produtividade real da cultura, já que é uma das localidades que apresenta uma das maiores PPf (6.638 $\left.\mathrm{kg} \mathrm{ha}^{-1}\right)$.

Os resultados de EC obtidos por municípios para a cultura da soja condizem com os dados apresentados por CUNHA et al. (2001a), que identificaram que as áreas mais propícias à ocorrência de déficit hídrico no estado do Rio Grande do Sul são aquelas situadas nas regiões oeste e sul, representadas 


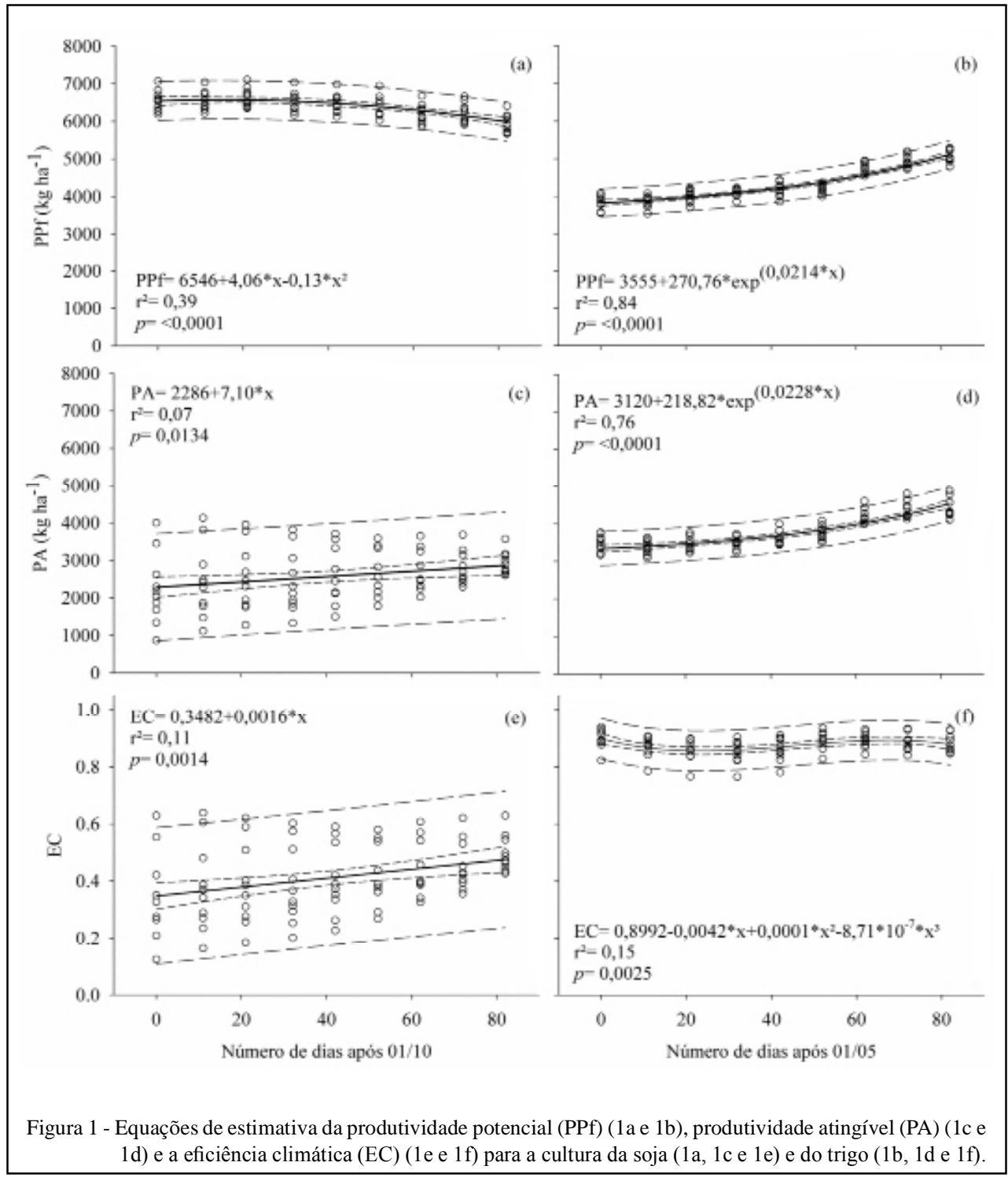

por São Gabriel, e as de menor probabilidade a leste e norte, representadas por Erechim. Por meio da relação entre a EC e o número de dias após a 01/10, observouse que há um ganho linear de eficiência da ordem de 0,0016 (Figura 1e) para cada dia de atraso na semeadura para a cultura da soja, o que indica que semeaduras tardias resultam em menores déficits hídricos durante o ciclo da cultura e, portanto, maior produtividade.

Para a cultura do trigo, observa-se que a maior PPf foi obtida no município de Erechim, que chegou a alcançar em média $4.478 \mathrm{~kg} \mathrm{ha}^{-1}$, sendo esse valor estatisticamente igual aos obtidos nos municípios de Júlio de Castilhos, São Borja e Veranópolis (Tabela 2). SILVA et al. (2010), trabalhando com diferentes genótipos de trigo em condições irrigadas, obtiveram produtividade média de dois anos de cultivo entre 4.808 e $6.655 \mathrm{~kg} \mathrm{ha}^{-1}$ para a localidade de Mococa, SP, resultado este dentro da ordem de grandeza observada neste trabalho.

Ao contrário do observado para a cultura da soja, para a do trigo houve concordância entre os valores de PPfe de PA para os decêndios de semeadura, pois ambas apresentaram ajuste exponencial quando relacionadas ao número de dias após 01/05 (Figura $1 \mathrm{~b}$ e 1d). Em função desse ajuste, a maior produtividade foi obtida em 20/07, no final da janela de semeadura. Os valores de PA do trigo variaram de 3.569 a $4.109 \mathrm{~kg} \mathrm{ha}^{-1}$ (Tabela 2), respectivamente, para São

Ciência Rural, v.43, n.3, mar, 2013. 
Borja e Erechim. Esses resultados são superiores à média observada no Rio Grande do Sul, que, segundo o IBGE (2010), está entre 1.104 e $2.106 \mathrm{~kg} \mathrm{ha}^{-1}$, mostrando que o déficit hídrico não é o principal fator que afeta a produtividade do trigo no estado. Isso também foi observado por ALBERTO et al. (2006), os quais constataram que o déficit hídrico em Santa Maria, RS, é maior para culturas de verão do que para as de inverno.

Para a cultura do trigo, verificou-se diferença significativa da EC entre as localidades de cultivo (Tabela 2). Os maiores valores de EC foram observados em Erechim $(0,92)$, Passo Fundo $(0,92)$, Júlio de Castilhos $(0,90)$ e Encruzilhada do Sul $(0,89)$, os quais foram estatisticamente semelhantes entre si, enquanto que o pior desempenho foi obtido em São Borja, com EC média de 0,81 , porém ainda elevado, mostrando que a perda média devido ao déficit hídrico é inferior a $20 \%$. A relação entre a EC do trigo e o número de dias após 01/05 apresentou ajuste cúbico, com significância a 5\%, obtendo maiores valores no início do período de semeadura (01/05), com estabilização no meio da janela de semeadura e decaindo ao final do período de semeadura analisado. Considerando-se que a perda de produtividade do trigo devido ao déficit hídrico é baixa, a data preferencial de semeadura para essa cultura deve seguir os valores de PPf mais elevados. Isso ocorre nas semeaduras realizadas a partir de meados de junho e durante o mês de julho.

\section{CONCLUSÃO}

Os resultados do presente trabalho permitiram concluir que o atraso da semeadura da cultura da soja proporciona maiores produtividades devido ao aumento da EC. Para a cultura do trigo, as perdas de produtividade devido ao déficit hídrico são menores que as observadas na cultura da soja, o que permite recomendar as semeaduras tardias, entre meados de junho e final de julho, quando a produtividade potencial do trigo é maior, o que, como consequência, leva a maiores produtividades atingíveis.

\section{REFERÊNCIAS}

ALBERTO, C.M. et al. Água no solo e rendimento do trigo, soja e milho associados ao ElNiño Oscilação Sul. PesquisaAgropecuária Brasileira, v.41, p.1067-1075, 2006. Disponível em: <http:// dx.doi.org/10.1590/S0100-204X2006000700001>. Acesso em: 05 dez. 2011. doi: 10.1590/S0100-204X2006000700001.

BATTISTI, R. et al. Eficiência agrícola da produção de soja, milho e trigo no estado do Rio Grande do Sul. Ciência Rural, v.42, p.24-30, 2012. Disponível em: <http://dx.doi.org/10.1590/S0103-
84782012000100005>. Acesso em: 04 fev. 2012. doi: 10.1590/ S0103-84782012000100005.

BERGAMASCHI, $\mathrm{H}$. et al. Maize yield and rainfall on different spatial and temporal scales in Southern Brazil. Pesquisa Agropecuária Brasileira, v.42, p.603-613, 2007. Disponível em: <http://dx.doi.org/10.1590/S0100-204X2007000500001>. Acesso em: 03 nov. 2011. doi: 10.1590/S0100-204X2007000500001.

BERLATO, M.A. et al. Evapotranspiração máxima da soja, relações com a evapotranspiração calculada pela equação de Penman, evaporação de tanque "classe A" e radiação solar global. Agronomia Sulriograndense, v.22, p.243-259, 1986.

BERLATO, M.A.; FONTANA, D.C. El Niño e La Niña: impactos no clima, na vegetação e na agricultura do Rio Grande do Sul; aplicações de previsões climáticas na agricultura. Porto Alegre: UFRGS, 2003. 110p.

BONNECARRÈRE, R.A.G. et al. Estimativa das produtividades potencial e deflacionada da cultura de milho no Estado do Rio Grande do Sul em função das condições climáticas. Revista Brasileira de Agrometeorologia, v.15, p.280-288, 2007.

CUNHA, G.R. et al. Zoneamento agrícola e época de semeadura para a soja no Rio Grande do Sul. Revista Brasileira de Agrometeorologia, v.9, p.446-459, 2001a.

CUNHA, G.R. et al. Zoneamento agrícola e época de semeadura para trigo no Brasil. Revista Brasileira de Agrometeorologia, v.9, p.400-414, 2001 b.

DOORENBOS, J.; KASSAM, A.M. Efeito da água no rendimento das culturas. Campina Grande: FAO, 1994. (Estudos FAO, Irrigação e Drenagem 33).

IBGE (INSTITUTO BRASILEIRO DE GEOGRAFIA E ESTATÍSTICA). Produção agrícola municipal. Rio de Janeiro, 2010. Acesso em: $01 \mathrm{dez}$. 2011. Online. Disponível em: <http:// www.ibge.gov.br>.

MAPA (MINISTÉRIO DA AGRICULTURA, PECUÁRIA E ABASTECIMENTO). Portarias de zoneamento agrícola de risco climático. Acesso em: 11 jan. 2012. Online. Disponível em: <http:// www.agricultura.gov.br/politica-agricola/zoneamento-agricola〉.

MOREIRA, M.A. et al. Eficiência do uso da radiação e índice de colheita em trigo submetido a estresse hídrico em diferentes estádios de desenvolvimento. Scientia Agricola, v.56, p.597603, 1999. Disponível em: <http://dx.doi.org/10.1590/S010390161999000300012>. Acesso em: 21 out. 2011. doi: 10.1590/ S0103-90161999000300012.

OSÓRIO, E.A.; WENDT, W. Duração do período de formação do grão em trigo. Scientia Agricola, v.52, p.395-398, 1995. Disponível em: <http://dx.doi.org/10.1590/S010390161995000200031>. Acesso em: 18 set. 2011. doi: 10.1590/ S0103-90161995000200031.

PEREIRA, A.R. et al. Agrometeorologia: fundamentos e aplicações práticas. Guaíba: Agropecuária, 2002. 478p.

RODRIGUES, O. et al. Efeito do fotoperíodo e da temperatura do ar no desenvolvimento da área foliar em soja (Glycine 
max L.). Passo Fundo: Embrapa Trigo, 2006. 27p. (Boletim de Pesquisa e Desenvolvimento, 33).

SILVA, A.H. da et al. Potencial de genótipos de trigo duro para produtividade e caracteres agronômicos no estado de São Paulo. Bragantia, v.69, p.535-546, 2010. Disponível em: <http://dx.doi. org/10.1590/S0006-87052010000300004>. Acesso em: $15 \mathrm{dez}$. 2011. doi: 10.1590/S0006-87052010000300004.
THORNTHWAITE, C.W.; MATHER, J.R. The water balance. Nex Jersey: Drexel Institute of Technology, $1955.104 \mathrm{p}$. (Publications in Climatology).

VENTIMIGLIA, L.A. et al. Potencial de rendimento da soja em razão da disponibilidade de fósforo no solo e dos espaçamentos. Pesquisa Agropecuária Brasileira, v.34, p.195199, 1999. Disponível em: <http://dx.doi.org/10.1590/S0100204X1999000200007>. Acesso em: 20 dez. 2011. doi: 10.1590/ S0100-204X1999000200007.

Ciência Rural, v.43, n.3, mar, 2013. 